\title{
Análise conjunta com estimulação em duas etapas
}

\author{
Marcelo Battesini \\ Mestre em Engenharia \\ Programa de Pós-Graduação em Engenharia de Produção - UFRGS \\ battesini@ig.com.br \\ Carla S. ten Caten \\ Doutora em Engenharia \\ Programa de Pós-Graduação em Engenharia de Materiais - UFRGS \\ tencaten@ppgep.ufrgs.br
}

Em uma pesquisa de Análise Conjunta, são utilizados conceitos de Pesquisa de Marketing, Projeto de Experimentos e Análise de Regressão. A utilização destes conceitos no planejamento da Análise Conjunta com Estimulação em Duas Etapas (AC com E2E), aliada à incorporação da perspectiva dos respondentes, busca aproximar a tarefa dos respondentes à situação real de compra, sem reduzir a quantidade de informação obtida com a pesquisa. Para isto, a estimulação utilizada na coleta de dados é flexível o suficiente para acomodar e propiciar o uso dos diferentes modelos de decisão adotados pelos respondentes. O método é ilustrado através de uma pesquisa sobre o mestrado profissionalizante oferecido pela Escola de Engenharia da Universidade Federal do Rio Grande do Sul. Esta aplicação da AC com E2E permitiu obter um modelo de preferência confiável que foi validado por uma amostra de controle, o que consolida o método proposto.

Palavras-chave: análise conjunta; comportamento do consumidor; pesquisa de marketing.

Conjoint Analysis research usually uses concepts of Marketing Research, Design of Experiments and Regression Analysis. The utilization of those concepts in planning of Conjoint Analysis with Two Stages Stimulation (AC with E2E), allied to the incorporation of respondents' perspective, searches to approximate the respondents' task to real purchase situation, without reducing the information amount obtained with the research. For this, the stimulation used in data collection is flexible enough to accommodate and to propitiate the use of different decision models adopted by the respondents. The method is illustrated through a research on the vocational master's degree offered by the Engineering Graduate Program of the Federal University of Rio Grande do Sul. The use of AC with E2E allowed obtaining a trustful preference model that was validated by a control sample, which consolidates the proposed method.

Keywords: conjoint analysis; consumer behaviour; marketing research.

\section{Introdução}

Segundo Miller et al. (1998), a criação de valor para os consumidores é a chave do sucesso de qualquer organização voltada para o mercado. Uma empresa tem por objetivo oferecer um produto ou serviço, de maneira diferente e melhor, para que o mercado-alvo venha a preferi-lo e até mesmo pagar um melhor preço por ele (KOTLER, 1999). Neste contexto, um importante diferencial gerencial é determinar quais produtos ou serviços são valorizados pelos consumidores, bem como qual é o valor que eles estão dispostos a pagar pelos mesmos.

A Análise Conjunta (AC), também conhecida como Conjoint Analysis, State of Preference, Planejamento
Conjunto, Análise Paritária, Preferência Declarada ou Trade-Off Analysis é uma ferramenta de auxílio na modelagem das decisões tomadas pelo ser humano. Segundo Intelliquest, s.d., a AC está baseada na hipótese que as complexas decisões, como as de compra, dependem não apenas de um único e sim de múltiplos atributos. Em AC, a preferência do consumidor é decomposta a partir da avaliação de um conceito global de produto ou serviço. Esta abordagem é mais realista que a utilizada em pesquisas de marketing tradicionais, pois está baseada na avaliação conjunta das diversas dimensões do produto ou serviço.

Mas afinal, o que é a Análise Conjunta?

A AC pode ser definida como um experimento, em função das ferramentas que utiliza em seu planejamento, 
e também como uma pesquisa de marketing, em função de seu objetivo de estimar a preferência do consumidor. Como são utilizados conhecimentos de Pesquisa de Marketing e de Projeto de Experimentos, os autores deste trabalho acreditam que a AC pode ser caracterizada como uma pesquisa experimental inserida no contexto de pesquisa de marketing, ou resumidamente como uma pesquisa de marketing.

Segundo Intelliquest (s.d.), a AC é uma vasta e mal definida família de técnicas, que têm em comum a modelagem estatística das avaliações dos respondentes, em relação aos cenários experimentalmente projetados. De acordo com Churchill e Nielsen (1996), a palavra "conjunta" tem a ver com a noção de que, quando os atributos são considerados conjuntamente, seu valor relativo pode ser mensurado com uma intensidade que não é possível quando eles são questionados um por vez. A AC é definida na literatura como: um experimento aplicado às decisões de marketing (GUSTAFSSON et al., 1999); uma ferramenta estatística de apoio às decisões de marketing, geralmente utilizada na etapa de desenvolvimento e teste de conceito de produtos (KOTLER, 2000); uma técnica multivariada utilizada especificamente para compreender como os respondentes desenvolvem suas preferências (HAIR et al., 1998); como uma família de procedimentos de pesquisa utilizada para planejar e analisar experimentos (MALHOTRA, 2001); ou como uma metodologia integrada de técnicas de coleta de dados, planejamento experimental e procedimentos de modelagem (LEE e GEISTFELD, 1998).

Segundo Battesini e Caten (2001), a AC é uma Pesquisa de Marketing que utiliza conceitos de Projeto de Experimentos e Análise de Regressão para planejar, coletar, analisar, realizar simulações e apresentar os resultados relevantes sobre o comportamento do consumidor, em relação a uma situação específica de marketing. Em $\mathrm{AC}$ os respondentes realizam um trade-off (relação de compromisso) ao avaliar produtos ou serviços, reais ou hipotéticos, normalmente representados por um conjunto planejado de atributos. A partir das avaliações dos respondentes é gerado um modelo de preferência, capaz de representar o comportamento do consumidor.

O desafio que se apresenta ao pesquisador no planejamento de uma pesquisa de AC é aproveitar ao máximo as ferramentas de Pesquisa de Marketing e Projetos de Experimentos, de forma a reduzir a complexidade e a quantidade de avaliações (esforço do respondente) sem diminuir a capacidade de modelar seu comportamento. O domínio dos conhecimentos utilizados nas diversas etapas de uma pesquisa de AC é um importante diferencial, para que o pesquisador consiga perceber o impacto que as decisões tomadas durante o planejamento terão na etapa da coleta de dados. A literatura apresenta várias abordagens (métodos ou técnicas) para superar este desafio.
Os métodos de AC variam grandemente em como elas são planejadas, administradas e modeladas, sem que exista nenhuma técnica única adequada a todas aplicações. Existem algumas variações na forma como os autores se referem, denominam e apresentam a AC. Hair et al. (1998) consideram basicamente três metodologias de AC: a Traditional Conjoint Analysis, Adaptive ou Hybrid Conjoint Analysis (ACA) e Choice-Based Conjoint Analysis (CBCA). Enquanto que Intelliquest (s.d.) considera 06 tipos (técnicas) de AC: Traditional FullProfile Conjoint Analysis, Adaptive Conjoint Analysis, CBCA, Partial Profile Choice Experiments, Availability Experiments e Best/Worth Conjoint Analysis. Já para Green e Srinivasan (1990) a Self-Explicated method (SEM), a Hybrid Conjoint Analysis (HCA) e a ACA seriam mais apropriadamente conceituados como métodos de mensuração das estruturas de preferência, em um modelo de intenção ou previsão de escolha.

O objetivo deste artigo é propor um método para o planejamento de pesquisas de Análise Conjunta com Estimulação em Duas Etapas (AC com E2E). O objetivo do método é propor uma forma de estimulação que aproxime a tarefa dos respondentes a uma situação real de compra atenuando o esforço dos respondentes, sem reduzir a capacidade de modelar as preferências dos consumidores. O método é ilustrado por meio de uma pesquisa sobre o Mestrado Profissionalizante oferecido pela Escola de Engenharia da Universidade Federal do Rio Grande do Sul, apresentado em Battesini (2002).

\section{Método para o planejamento de uma pesquisa de análise conjunta com estimulação em duas etapas}

Alguns dos erros comuns em Pesquisas de Marketing são causados por deficiências em seu planejamento ou concepção. Boyd et al. (1989) e Kotler (2000) sugerem o uso do método científico em pesquisas de marketing. Em função de suas características, uma pesquisa de AC deve ser cuidadosamente planejada. Neste sentido, a estrutura de etapas de uma pesquisa de AC deve ser compatível com as do Método Científico, de uma Pesquisa de Marketing e de um Planejamento Experimental (ver comparação na Figura 1). Esta compatibilidade pode ser obtida a partir da integração das etapas, já estabelecidas na literatura, das duas áreas de conhecimento que são a base de qualquer pesquisa de AC: Pesquisa de Marketing e Projeto de Experimentos. Com isto é gerado um método consistente com os métodos de AC apresentados na literatura.

Desta forma, o método proposto incorpora as cinco macro-etapas propostas por Kotler (2000) para uma pesquisa de marketing, e através da sub-divisão destas, as etapas propostas por Montgomery (1976) para um 


\begin{tabular}{|c|c|c|c|}
\hline Etapa & Método Científico & Pesquisa de Marketing & Experimento \\
\hline 1 & Definição do objeto de observação & - & - \\
\hline 2 & Observação do problema & $\begin{array}{l}\text { Definição do problema e dos objetivos } \\
\text { da pesquisa }\end{array}$ & $\begin{array}{l}\text { Identificação e estabelecimento } \\
\text { do problema }\end{array}$ \\
\hline \multirow{3}{*}{3} & \multirow{3}{*}{ Formulação das hipóteses } & \multirow{3}{*}{ Desenvolvimento do plano de pesquisa } & Seleção da variável de resposta \\
\hline & & & Escolha dos fatores e seus níveis \\
\hline & & & Escolha do projeto experimental \\
\hline 4 & Experimentação & Coleta das informações & Execução do experimento \\
\hline 5 & Análise dos resultados experimentais & Análise das informações & Analise dos dados \\
\hline 6 & Conclusão sobre a validade das hipóteses & Apresentação das conclusões & Conclusões e recomendações \\
\hline 7 & Generalização a respeito das conclusões & - & - \\
\hline
\end{tabular}

Adaptado de Kotler (2000) e Montgomery (1976).

Figura 1 - Etapas do Método Científico, de uma Pesquisa de Marketing e de um Experimento

planejamento experimental. As etapas resultantes, apresentadas na Figura 2, são interativas e as definições adotadas a cada etapa afetam as seguintes.

A estruturação das etapas posiciona a AC com E2E como uma Pesquisa de Marketing, e não como uma ferramenta estatística ou como uma técnica de dependência, como muitas vezes é apresentado na literatura. Assim, o objetivo de inserir nas etapas de uma Pesquisa de Marketing as de um Projeto de Experimental é caracterizar a AC com E2E, e também a AC, como uma Pesquisa de Marketing que utiliza conhecimentos de Projeto de Experimentos como ferramenta para planejar a coleta e análise dos dados.

A lógica utilizada na construção da AC com E2E permite o uso de suas etapas em outros métodos de AC. Como é possível estabelecer uma sequiência de etapas genérica aos diversos métodos de $\mathrm{AC}$, a diferença entre métodos não está na sequiência de etapas utilizada. A diferença entre os diversos métodos de AC ocorre especificamente na etapa de definição da estimulação da coleta de dados (ver item 2.2.3) e conseqüentemente se reflete na etapa de análise das informações (ver item 2.4). A partir deste entendimento, pode-se afirmar que a AC com E2E se caracteriza como um método ao estabelecer uma seqüência de etapas, mas principalmente ao estabelecer uma específica forma de estimulação para a coleta de dados.

As pesquisas de AC devem ser planejadas levando em conta a perspectiva do respondente, para permitir que os respondentes utilizem modelos de escolha reais ou o mais próximo possível, de forma que o consumidor não precise alterar substancialmente seu comportamento de compra ao responder a pesquisa. A situação ideal de pesquisa seria aquela onde a estimulação utilizada na

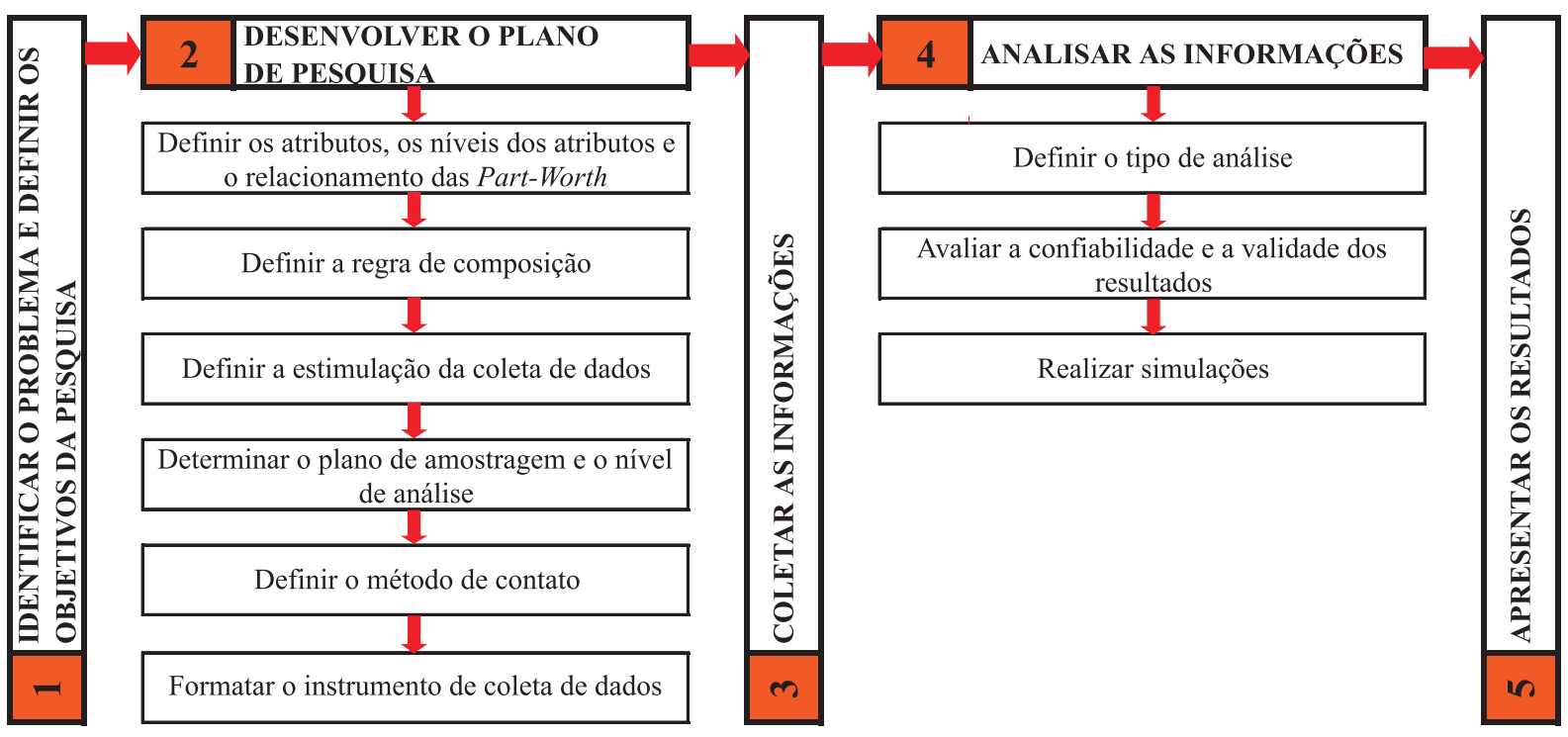

Figura 2 - Etapas de uma pesquisa de AC com E2E. 
pesquisa fosse similar àquela utilizada pelos consumidores no momento da compra. Como normalmente o modelo de decisão a ser adotado por cada consumidor é desconhecido, esta situação ideal é de difícil implementação. Mesmo que o pesquisador conheça previamente os modelos de decisão, seria necessário planejar estimulações distintas para os diferentes respondentes, o que dificultaria excessivamente o planejamento da pesquisa.

O planejamento de uma pesquisa de AC com E2E procura minimizar esta situação ao propor uma estimulação flexível o suficiente para acomodar os diferentes modelos de decisão adotados pelos respondentes. A AC com E2E está baseada nas seguintes premissas:

(i) na lógica utilizada na estruturação de suas de etapas;

(ii) na estimulação da coleta de dados em duas etapas (item 2.2.3.1);

(iii) no uso da blocagem para reduzir a uma quantidade razoável o número total de cenários a serem avaliados pelo respondente, para gerar um grupo de cenários equilibrados (ortogonais) garantindo um ambiente competitivo entre eles (item 2.2.3.1);

(iv) na redução, em relação ao total de cenários planejados experimentalmente, do número de avaliações formalmente realizadas pelo respondente utilizando uma escala de medida (item 2.2.3.2);

(v) e na modelagem das preferências a partir apenas das avaliações formalmente realizadas na segunda etapa da estimulação (item 2.2.3.2).

\subsection{Identificar o problema e definir os objetivos da pesquisa}

Uma vez estabelecido o problema de marketing, é preciso definir o problema de pesquisa e identificar com precisão o seu objetivo. Para isto, o pesquisador deve compreender a situação específica de marketing na qual o problema de marketing está inserido. Segundo Yin (1994), é necessário tempo e paciência para a definição da questão da pesquisa, uma vez que este é provavelmente o mais importante passo a ser dado em uma pesquisa.

A delimitação do problema de pesquisa pode envolver, além dos pesquisadores, os representantes das diversas áreas da empresa, tais como: desenvolvimento de produto, marketing, vendas, administração e produção. Nesta etapa, o pesquisador deve preliminarmente investigar as características da população a serem considerados no plano de amostragem, tais como, a homogeneidade da população e a existência de estratos.

O objetivo geral da AC é estimar um modelo de preferência, baseado nas importâncias de cada atributo, cal- culadas através das respostas dadas pelos respondentes aos cenários apresentados (EVRARD et al., 1993). A obtenção de um modelo válido de comportamento do consumidor permite o desenvolvimento de inúmeras estratégias gerenciais, tais como a definição de combinações ótimas de atributos, a mensuração da importância relativa dos atributos, a otimização e o desenvolvimento de conceito de produtos e serviços, a identificação de tamanhos de mercado ou a mensuração de elasticidade de preço. A definição em relação à estratégia gerencial a ser adotada deve ocorrer nesta etapa, para que seja possível um apropriado planejamento da pesquisa.

\subsection{Desenvolver o plano de pesquisa}

Nesta etapa o pesquisador já conhece o produto ou serviço objeto da pesquisa, devendo então definir as estratégias a serem adotadas para obtenção das informações. É na etapa de desenvolvimento do plano de pesquisa que o pesquisador irá utilizar seus conhecimentos de AC, para a obtenção dos dados necessários à solução do problema de marketing.

\subsubsection{Definir os atributos, os níveis dos atributos e o relacionamento das Part-Worth}

Apesar da definição dos atributos, e de seus níveis, não ser tão complexa quanto as demais etapas do planejamento, ela é uma das mais importantes de uma pesquisa de AC. Os atributos selecionados devem ser capazes de representar o objeto de pesquisa, além de influenciar a decisão de compra do consumidor. A incorreta seleção dos atributos pode determinar resultados não válidos, e desta forma, não adequados aos propósitos inicialmente estabelecidos.

Para identificar os atributos, podem ser utilizadas pesquisas exploratórias em profundidade como os grupos focados (CHURCHILL e NIELSEN, 1996 e MALHOTRA, 2001), pesquisas descritivas conclusivas (GREEN e KRIEGER, 1991), a experiência do contratante aliada à do pesquisador (GUSTAFSSON et al., 1999 e MALHOTRA, 2001) ou a realização de um piloto seguido da pesquisa de AC (HAIR, 1998 e MALHOTRA, 2001).

Em alguns casos, o conhecimento do contratante aliado ao do pesquisador são suficientes para selecionar os atributos e seus níveis. Na maioria das vezes, este raciocínio é uma soberba que pode levar a resultados incorretos. Idealmente, uma pesquisa de AC deveria ser precedida de duas etapas (pesquisas): uma etapa qualitativa, para definir atributos, e uma etapa quantitativa, para selecionar os atributos representativos e os seus níveis. Quando os pesquisadores não têm disponibilidade de tempo ou de recursos para adotar esta recomendação, deve pelo menos ser realizada uma pesquisa qualitativa, de forma que o input para a definição dos atributos e 
de seus níveis seja baseado em demandas do mercado consumidor. Nestes casos, outro recurso, que não a pesquisa quantitativa, deve ser utilizado para seleção dos atributos gerados pela pesquisa qualitativa.

A seleção dos níveis dos atributos pode ser determinada pelas próprias características dos atributos (apenas dois níveis, presença ou ausência) ou serem especificados pelo pesquisador de modo a investigar: uma faixa de variação dos atributos $(50 \mathrm{~g}, 75 \mathrm{~g}$ e $100 \mathrm{~g})$, quando eles são métricos ou de níveis específicos, quando eles são não-métricos (pequeno, médio e grande).

Uma vez definidos os atributos, o pesquisador deve estabelecer como será o relacionamento entre os seus níveis. Os relacionamentos entre os níveis dos atributos são estimados através das Part-Worth ou Part-Worth Utilities, que são as estimativas geradas em AC para as preferências, ou utilidades, associadas a cada nível dos atributos. Segundo Green e Srinivasan (1990), em termos da forma da função matemática, os relacionamentos entre as Part-Worth podem ser Linear ou Vetorial, Quadrático ou Ideal ou então de Part-Worth independentes. O mais restritivo é o Linear, onde é estimada apenas uma Part-Worth, que é utilizada para todos os níveis do atributo, como ocorre com os coeficientes em uma equação de regressão. O menos restritivo é o de Part-Worth independentes, onde é estimada uma diferente Part-Worth para cada nível do atributo.

O tipo de relacionamento entre as Part-Worth pode ser estipulado individualmente para cada atributo. Desta forma, é possível que apenas um atributo de interesse assuma um relacionamento tipo Part-Worth independentes e os demais relacionamentos Lineares. As estimativas são mais eficientes e confiáveis, sob o ponto de vista estatístico, quando é possível reduzir o número de atributos com relacionamentos de Part-Worth independentes (HAIR et al., 1998).

A investigação de relacionamentos do tipo Part-Worth independentes pode ser útil, quando o efeito dos níveis dos atributos se comporta de forma instável (crescente e decrescente). Desta forma, a capacidade de estimação do modelo pode ser melhorada, desde que existam graus de liberdade suficientes. Esta definição deve ocorrer antes da etapa de definição da estimulação da coleta de dados, uma vez que o número de parâmetros a ser estimado interfere no nível de fracionamento do planejamento experimental (item 1.2.3.1).

Em alguns casos, pode ser necessária a utilização de atributos mantidos constantes em todos os cenários, para que os mesmos não interfiram no resultado da pesquisa. Por exemplo, em uma pesquisa sobre uma televisão, onde os quatro atributos selecionados sejam a marca, o formato da tela, as condições de pagamento e o preço, os atributos: com controle remoto e com entrada para áudio poderiam ser apresentados constantes em todos os cenários apresentados aos respondentes.

Ao selecionar os atributos, o pesquisador deve tomar cuidado para não incluir atributos com níveis hierárquicos muito diferentes, em relação à sua capacidade de influenciar o comportamento do consumidor. No exemplo da televisão, caso fossem também incluídos os atributos Close Caption ou tecla SAP (presença ou ausência), eles possivelmente teriam menor influência sobre a decisão de compra de um consumidor médio, do que os demais.

O pesquisador precisa ter em mente que o método de contato, a forma de representar os atributos e o grau de conhecimento dos respondentes são componentes que interferem no número máximo de atributos a ser utilizado. Em pesquisas de $\mathrm{AC}$ onde as entrevistas são pessoais, os cenários são representados através de recursos pictoriais e a amostra é composta por indivíduos com grande conhecimento sobre o objeto da pesquisa, os respondentes têm condições de avaliar um número maior de atributos.

\subsubsection{Definir a regra de composição}

Os respondentes normalmente adotam estratégias para reduzir o seu esforço quando estão diante de um processo de decisão. Os modelos de escolha ou choice model se referem às específicas maneiras de como os consumidores selecionam uma, entre diversas alternativas. Existem duas grandes categorias de modelos de escolha: os compensatórios e os não-compensatórios (SHETH et al., 1999). Segundo Lee e Geistfeld (1998), nos modelos compensatórios admite-se que o consumidor considera todos os seus atributos ao avaliar uma dada alternativa, e desta forma, a presença ou ausência de um atributo pode ser compensada por outro. Já nos modelos nãocompensatórios, admite-se que os consumidores não realizam o trade-off de um atributo por outro, e desta forma, as alternativas são avaliadas de forma categórica, pela presença ou ausência dos atributos considerados mais importantes.

Com base no conhecimento adquirido nas etapas anteriores, nesta etapa o pesquisador deve estimar qual é a regra de composição mais adequada para gerar o modelo de preferência que representará o modelo de decisão utilizado pelos consumidores. Os pesquisadores podem adotar uma regra de composição simplesmente aditiva ou então também incluir termos de interação, para acomodar os diferentes modelos de decisão adotados pelos respondentes ao modelo de preferência a ser gerado.

Ao definir sobre a regra de composição, o pesquisador deve considerar alguns aspectos. O primeiro, é que os respondentes podem utilizar comportamentos compen- 
satórios ou não-compensatórios, em função do número de cenários a serem avaliados e da quantidade de tempo disponível para a pesquisa. O segundo, é que em função do seu grau de conhecimento sobre o objeto da pesquisa, os respondentes podem ter maior ou menor facilidade de realizar as avaliações. O terceiro, é a avaliação das características do produto ou serviço objeto da pesquisa, uma vez que sua natureza pode suscitar por parte dos respondentes avaliações emocionais, como quando é utilizado o atributo marca. Nestes casos, as interações ocorrem com maior frequiência. O quarto, é que ao definir a regra de composição, o pesquisador está determinando a quantidade de cenários a serem avaliados pelo respondente, e neste sentido, o seu esforço. Quando é utilizado o modelo aditivo é necessário um número menor de cenários para avaliar as Part-Worth, do que quando o modelo inclui termos de interação. Assim, a necessidade de avaliar efeitos de interação reduz o grau de fracionamento possível do número total de cenários.

\subsubsection{Definir a estimulação da coleta de dados}

A estimulação se refere ao sistema de medição utilizado na mensuração da preferência a ser modelada. A preferência do respondente representa a sua avaliação simultânea de vários aspectos (dimensões) associados ao produto ou serviço. Ao definir a estimulação, é necessário que o pesquisador compreenda qual o seu impacto nas etapas seguintes, uma vez que a coleta e a análise dos dados ficarão condicionadas a estas decisões.

Especialmente nesta etapa, o pesquisador deve ter em mente a perspectiva do respondente, já que estará definindo o nível de complexidade das avaliações do respondente (tipo e número de cenários a serem avaliados) que influencia a consistência (confiabilidade) das respostas. Uma das limitações das pesquisas de AC é que o respondente pode ter dificuldade, ou até mesmo se sentir incapaz de avaliar as inúmeras alternativas a ele apresentadas. Conforme aumenta a complexidade das avaliações a que são submetidos, ocorre uma sobrecarga dos respondentes, o que pode diminuir a confiabilidade das respostas.

A simplificação da coleta de dados é o desafio que se apresenta aos pesquisadores, no planejamento de uma pesquisa de AC. As possibilidades de planejamento experimental são inúmeras e dependem do número de atributos, de seus níveis e da regra de composição adotada (grau de fracionamento). A simples transposição dos cenários de um planejamento experimental para uma pesquisa de AC gera a demanda de um elevado número de avaliações por parte dos respondentes, mesmo que com um bom nível de fracionamento ou blocagem. Por exemplo, em um planejamento experimental do tipo $2^{7-2}$ são necessárias 32 avaliações, correspondentes a uma avaliação por cenário, ou seja, o respondente avalia cada um dos cenários individualmente e de forma descontextualizada do todo. Esta abordagem não reconhece a capa- cidade do respondente em visualizar diversos cenários simultaneamente e realizar apenas uma escolha entre eles. Desta forma, o pesquisador deve considerar a capacidade de avaliação dos respondentes, ou seja, os modelos de decisão utilizados, ao invés de propor a obtenção de um grande número de avaliações individuais descontextualizadas do todo, de modo a satisfazer conceitos estatísticos.

Para caracterizar a importância da estimulação da coleta de dados em $\mathrm{AC}$ com E2E, as etapas que se referem às definições em relação à complexidade das avaliações a serem realizadas pelos respondentes foram agrupadas. A estimulação a ser utilizada na coleta de dados envolve a definição da combinação dos atributos para a composição dos cenários, da escala de resposta e da forma de representação dos cenários.

\subsubsection{Definir a combinação dos atributos para a composição dos cenários}

Esta é uma das etapas mais trabalhosas de uma pesquisa de AC, juntamente com a análise dos resultados, sendo comum o uso de rotinas computacionais. Nesta etapa, são utilizados conhecimentos de Projeto de Experimentos para identificar as combinações dos níveis dos atributos que irão compor os cenários; neste sentido, alguns autores comparam a $\mathrm{AC}$ a planejamento experimental.

A literatura de Projetos de Experimentos apresenta inúmeras formas de planejamento experimental, não sendo objetivo deste artigo esgotá-las. Em AC com E2E, o planejamento experimental dos cenários é feito assim como em Análise Conjunta Tradicional, e desta forma, a decomposição das Part-Worth é conduzida a partir das avaliações de cenários do tipo Full Profile (que representam produtos ou serviços completos). Normalmente, os cenários são criados utilizando conceitos de ortogonalidade, e com isso o impacto de cada atributo na variável de resposta (preferência) pode ser mensurado independentemente.

Em geral é necessário um número relativamente grande de atributos para definir um produto ou serviço. $\mathrm{O}$ aumento neste número produz um crescimento geométrico no número de possibilidades de combinação. Quando o número de combinações não é factível, em função do esforço que seria demandado ao respondente, pode ser necessário utilizar técnicas de fracionamento e/ou de blocagem. Deve-se notar o planejamento experimental (fracionamento e blocagens para gerar a matriz experimental) utilizado para a criação dos cenários deve considerar a regra de composição adotada, ou seja, se é ou não necessário estimar as interações.

Em AC com E2E, o fracionamento normalmente é conduzido de forma a garantir o cálculo dos efeitos principais e dos efeitos de interações que se deseja 
conhecer. Geralmente, o pesquisador pode admitir uma perda da capacidade de avaliar os efeitos de interações de mais alta ordem (entre mais de dois atributos), em favor da redução do número total de cenários a serem avaliados. Já a blocagem tem três objetivos:

- possibilitar o envolvimento de mais de um respondente na tarefa de avaliação do número total de cenários da matriz experimental. Desta forma, um respondente pode avaliar os cenários de um ou de diversos blocos, dependendo do planejamento experimental. $\mathrm{O}$ uso de blocos equilibrados de cenários (com igual número de níveis de atributos) permite o cálculo independente das Part-Worth, mesmo que o número total de questionários da matriz experimental tenha sido respondido por mais de um respondente;

- possibilitar a redução, em relação ao número total de cenários a serem avaliados por um mesmo respondente, para conjuntos menores (blocos) e mais facilmente manuseáveis;

- garantir um ambiente competitivo entre cenários (blocos). A estimulação em duas etapas e o uso de blocos onde os cenários competem entre si pela preferência do respondente aproxima a situação de pesquisa àquela utilizada em processos de decisão de situações reais.

Assim, a estimulação utilizada em AC com E2E é viabilizada pelo uso da blocagem e possui as seguintes características:

- na primeira etapa, os respondentes escolhem um entre os diversos cenários de cada bloco, assim como ocorre na situação real de compra, onde o consumidor geralmente seleciona apenas um entre vários produtos ou serviços;

- na segunda etapa, os respondentes avaliam os cenários escolhidos na primeira etapa, através de alguma escala de medida (ver item 2.2.3.2). Assim, o número de blocos determina o número de cenários escolhidos na primeira etapa, ou seja, aqueles que o respondente deverá avaliar utilizando a escala de medida na segunda etapa.

Desta forma, a estratégia de blocagem influencia no esforço de avaliação dos respondentes. Enquanto que na primeira etapa da estimulação o respondente apenas escolhe um dos cenários do bloco, na segunda ele realiza uma avaliação refinada (mais precisa) e formal, apenas dos cenários previamente escolhidos.

A estimulação em duas etapas está baseada nos modelos de escolha dos consumidores. Ela propicia ao respondente adotar um comportamento não-compensatório (primeira etapa), para na segunda etapa adotar um com- portamento compensatório, ou até mesmo manter um comportamento não-compensatório. Assim, o tempo dedicado pelo respondente é utilizado em sua maior parte para a avaliação dos cenários que potencialmente representariam as suas escolhas. Com isto, a estimulação utilizada em AC com E2E é flexível o suficiente para acomodar e propiciar o uso dos diferentes modelos de decisão adotados pelos respondentes, sejam elas compensatórias ou não.

Assim em AC com E2E, como em Análise Conjunta Baseada em Escolhas, na primeira etapa da estimulação os respondentes são estimulados apenas a escolher um entre um grupo de cenários (Choice Set), mas a escolha é realizada a partir de cenários do tipo Full Profile como em Análise Conjunta Tradicional.

Independentemente do planejamento experimental, cada respondente irá receber apenas um questionário, contendo o número total de cenários, ou uma fração dele. No caso de ser apresentado apenas uma parte dos cenários a cada respondente, o pesquisador precisa considerar o aumento necessário no número de respondentes em relação ao tamanho da amostra inicialmente calculada, para que seja avaliado o número mínimo de questionários completos. Por exemplo, caso o tamanho da amostra seja igual a 30 questionários e o questionário foi dividido em dois respondentes (2 blocos) são necessários 60 respondentes para obter 30 questionários completos.

\subsubsection{Definir a escala de medida da variável de resposta}

Como o modelo gerado deve ser capaz de representar as preferências dos consumidores, a variável de resposta em pesquisas de $\mathrm{AC}$ é a preferência dos respondentes pelos produtos ou serviços objeto da pesquisa. A escala de medida é um instrumento utilizado pelo pesquisador para converter as preferências do respondente em avaliações (variável de resposta). A preferência normalmente é expressa através de alguma escala de resposta, sendo que o instrumento de medição da variável de resposta é o próprio respondente.

Ao definir a escala de medida da variável de resposta, o pesquisador precisa ter em mente a perspectiva do respondente, uma vez que o tipo de variável de resposta adotado interfere no esforço ao qual o respondente será submetido, no realismo da situação de pesquisa e na análise dos dados.

Em AC com E2E a escala de medida utilizada depende da etapa da estimulação:

- na primeira etapa, o respondente realiza uma seleção preliminar, em cada bloco, através de uma simples escolha de um cenário (escala nominal 
(EO), variando do $1^{\circ}$ ao $16^{\circ}$, e de uma escala Intervalar (EI), variando de 0 a 100 pontos.

Caso exista consistência nas avaliações do respondente, a Figura 3 apresenta para o experimento PE II (quatro blocos) as respostas para a estimulação em duas etapas (AC com E2E). $\mathrm{Na} 1^{a}$ etapa as respostas estão expressas em uma escala nominal binária ( 0 ou 1 ), enquanto que na $2^{\mathrm{a}}$ etapa as respostas estão expressas em uma escala intervalar (0 a 100 pontos).

Pode-se observar que, dependendo da escala utilizada no PE I, o respondente é estimulado a realizar 16 ordenações (EO) ou 16 avaliações através da escala intervalar (EI). Já no PE II (AC com E2E) o respondente é estimulado a realizar quatro escolhas seguidas de quatro avaliações (total de oito avaliações). Desta forma, o número de avaliações realizadas através da estimulação em duas etapas (PE II) é menor do que em um experimento não-blocado (PE I). Sendo que a segunda etapa da estimulação permite que o respondente expresse com maior discernimento as suas preferências, em função do menor número de cenários.

Para ilustrar outros aspectos da estimulação em duas etapas, em relação ao comportamento do respondente, os cenários do PE II são apresentados na Figura 4, em ordem de preferência (EO). Os cenários com as melhores avaliações (C8, C16, C6 e C4) carregam as informações sobre os atributos que mais agradam ao respondente, 
mais adequado para os casos em que existe interesse de identificar apenas os produtos ou serviços com maior preferência, mas possui limitações quando o objetivo é de estudar, além destes, aqueles com preferências intermediárias.

Assim, em AC com E2E com o uso da blocagem e a avaliação apenas das melhores escolhas (segunda etapa), é possível reduzir a dificuldade da tarefa de formalmente avaliar todos os cenários, apesar dos respondentes considerarem todos em suas avaliações. Esta característica da estimulação da AC com E2E se estabelece a partir da construção de suas etapas, que considera a AC uma Pesquisa de Marketing. Nesta lógica, parece razoável abrir mão da necessidade de que todos os cenários sejam formalmente avaliados (necessidade estatística) de forma a aproximar a situação de pesquisa àquela real de tomada de decisão.

\subsubsection{Definir a forma de representação dos cenários}

As pesquisas de $\mathrm{AC}$ se diferenciam das demais formas de pesquisa por apresentarem simultaneamente diversas dimensões (atributos) do produto ou serviço objeto de pesquisa. Assim, quanto mais eficaz o método utilizado na representação dos cenários, melhor será a qualidade das avaliações dos respondentes, ao expressar as suas reais preferências. Em pesquisas de AC com E2E podem ser utilizadas as formas de representação verbais, por parágrafos ou pictoriais. Sempre que for possível a representação pictorial, devem ser utilizadas inclusive imagens e modelos reais.

Segundo Green e Srinivasan (1990), tem ocorrido um aumento do uso da descrição pictorial. Esta forma de representação torna a tarefa mais interessante para o respondente, além de ser uma forma menos ambígua de representar a informação, o que conseqüentemente aumenta o número de atributos que podem ser incluídos na pesquisa. $\mathrm{O}$ incremento no uso de materiais pictoriais tende a expandir o uso de $\mathrm{AC}$, pois aumenta o realismo na representação, ao replicar condições reais de mercado.

Quando não for possível representar integralmente o objeto de pesquisa através da representação pictorial, deve ser avaliada a possibilidade de utilizá-la parcialmente. Em alguns casos, podem ser utilizadas descrições por parágrafo associadas a representações pictoriais de uma parcela do total de atributos. 


\subsubsection{Determinar plano de amostragem e o nível de análise}

Deve ser dada especial atenção ao tipo e ao tamanho da amostra, uma vez que eles podem interferir na validade da pesquisa. Em AC com E2E sugere-se o uso de amostras probabilísticas. Segundo Churchill e Nielsen (1996), em amostras probabilísticas, para se determinar o tamanho da amostra representativa da população, devese considerar o desvio padrão da população (s), a precisão desejada (erro admitido para o resultado da pesquisa) e o grau de confiança (a) associado à pesquisa. O cálculo do tamanho de amostra, para os diversos tipos de amostragem, pode ser visto em Burns e Bush (1995), Churchill e Nielsen (1996) e Malhotra (2001).

Em AC com E2E a análise normalmente ocorre em nível agregado, mas ela pode ser conduzida em nível individual quando o número de avaliações formalmente realizadas pelos respondentes for suficiente para gerar um modelo de preferência. Segundo Hair et al. (1998), em Análise de Regressão Múltipla a regra geral é que a razão entre o número de avaliações e o número de variáveis independentes seja maior que 5 para 1 , apesar de o número desejado ser de 15 a 20 avaliações para 1 variável independente.

O número de entrevistas a serem conduzidas pode ser determinado em relação ao número de elementos da população ou ao tamanho de cada um dos seus estratos, dependendo do objetivo da pesquisa. Em AC, quando a amostra não é subdividida em estratos pode ser gerado um modelo agregado geral ou modelos individuais. A partir da análise em nível individual, podem ser posteriormente realizadas simulações com o objetivo de classificar os respondentes. Neste caso, pode ser utilizada a Análise de Aglomerados para estabelecer agrupamentos de respondentes a partir dos valores das Part-Worth de cada indivíduo. Já quando a amostra é subdividida em estratos, podem ser gerados modelos agregados para cada estrato, além de modelos individuais e agregados gerais. Em ambos casos é importante que nesta etapa o pesquisador identifique as variáveis de segmentação a serem registradas no questionário, uma vez que elas normalmente contribuem para a melhoria da validade dos modelos.

A blocagem pode ser utilizada para reduzir o número de cenários a ser avaliado por cada respondente, conseqüentemente o número de respondentes necessários para que se tenha o conjunto completo de cenários avaliados (matriz experimental) é aumentado na proporção do número de blocos utilizados. Em determinadas situações onde o número de elementos de um estrato é muito pequeno, a blocagem pode ter seu uso limitado, uma vez que podem não existir indivíduos suficientes para compor o questionário completo, dependendo do número de blocos.

\subsubsection{Definir o método de contato}

Em uma pesquisa de AC com E2E, podem ser utilizados métodos de contato telefônicos, postais e entrevistas pessoais. Nos casos onde o método de contato é telefônico ou postal, o pesquisador deve dar atenção especial em relação à complexidade do instrumento de coleta de dados. Um instrumento de coleta complexo pode ocasionar dificuldades no seu preenchimento, afetando a taxa de retorno e a confiabilidade da pesquisa, sendo recomendável a utilização do método Telefônico-PostalTelefônico.

Segundo Green e Surinivasan (1990), no método Telefônico-Postal-Telefônico todo o material da entrevista (questionários, cartões com cenários, brindes, etc.) é enviado pelo correio e os dados são posteriormente coletados por telefone, através de uma ligação previamente agendada. As questões mais simples são previamente respondidas, porém a parte do questionário com AC normalmente é reservada para ser respondida quando da ligação. Este método permite o uso de amostras probabilísticas, de materiais visuais (pictoriais), além de apresentar uma alta taxa (até 80\%) de retorno das informações e uma baixa taxa de falta de dados, uma vez que a maioria dos questionários é completamente respondida.

Sempre que possível deve ser dada preferência às entrevistas pessoais ou o uso da técnica do questionário, onde o próprio respondente preenche o questionário. Em ambos casos, através da presença do entrevistador, podem ser mais facilmente contornadas dúvidas em relação ao entendimento do instrumento de coleta de dados, uma vez que o respondente pode contar com o auxílio do entrevistador. Muitas vezes, o pouco tempo disponível e a falta de recursos para a pesquisa podem demandar uma revisão no método de contato e no plano de amostragem.

\subsubsection{Formatar o instrumento de coleta de dados}

O instrumento de coleta de dados ou questionário se refere a todos os elementos utilizados na coleta de dados da pesquisa. O questionário deve ser claro, conter todas as informações que serão utilizadas pelos respondentes, ser construído de forma a permitir uma consistente coleta das informações, bem como facilitar a consolidação dos dados.

Em AC com E2E os cenários são apresentados em questionários contendo as instruções e a escala de medida. Os cenários dos blocos podem ser apresentados em cartões individuais, desde que os blocos previamente estipulados sejam respeitados; porém, eles devem possuir clara identificação para facilitar o seu manuseio pelo respondente e possibilitar o correto registro das avaliações atribuídas pelo respondente junto ao questionário. 


\subsubsection{Avaliar a confiabilidade e validade} dos resultados

Em AC com E2E devem ser verificadas a qualidade do ajuste do modelo aos dados, adequação da regra de composição adotada, a Validade e a Confiabilidade dos resultados. Estas verificações servem para indicar a variabilidade existente nas respostas dadas pelos consumidores, que pode ocorrer de duas formas.

A primeira, é a variabilidade para um mesmo cenário que pode ocorrer para um mesmo respondente ou para diferentes respondentes. Para um mesmo respondente, ela ocorre pela falta de consistência das respostas de um mesmo respondente no tempo, ou seja, o respondente não realiza as mesmas avaliações para um mesmo cenário em momentos diferentes. Esta falta de estabilidade é prejudicial ao ajuste do modelo. Para diferentes res- 
onde:

- $\quad \mathrm{SQT}($ Soma Quadrada Total $)=\mathrm{SQReg}+\mathrm{SQR}$, sendo que SQReg é a Soma Quadrada da Regressão e SQR é a Soma Quadrada dos Resíduos;

- o termo (SQT - SQEP) representa a parcela da variação total presente na variável de resposta $y$ que pode ser explicada por algum modelo de regressão. 
utilizar as informações provenientes da pesquisa de AC com E2E para a tomada de decisão em relação ao problema de marketing.

\section{Aplicação de uma pesquisa de análise conjunta com estimulação em duas etapas}

Desta forma, em AC com E2E, o valor de $100 * \mathrm{R}^{2} / \mathrm{R}_{\text {máx }}^{2}$ (em percentual) é utilizado conjuntamente com a capacidade de prever as melhores respostas (percentual de acertos dos cenários preferidos) para a verificação da validade e do adequado ajuste do modelo.

\subsubsection{Realizar simulações}

A partir de um modelo com adequada validade podem ser realizadas simulações. As simulações podem ser conduzidas de três formas diferentes. A primeira é uma análise utilizando o modelo gerado a partir dos cenários submetidos aos respondentes para o cálculo das Preferências dos cenários que não foram avaliados pelos respondentes. Este procedimento possibilita identificar cenários viáveis e com valores de utilidade até mesmo maior que os inicialmente investigados.

A segunda é a comparação competitiva entre cenários. O contratante pode ter interesse em investigar o percentual de mercado ocupado por um ou vários produtos ou serviços (cenários). Normalmente, estas simulações são realizadas a partir de um questionamento do tipo: E se apenas os cenários A1, D4 e F3 fossem ofertados ao respondente, qual tamanho de mercado caberia a cada um deles? A terceira é a inserção de variáveis de segmentação no modelo, tais como: faixa etária, renda, escolaridade, classe social ou sexo. Este procedimento tende a melhorar a capacidade de previsão do modelo.

\subsection{Apresentar os resultados}

O pesquisador deve ter especial cuidado com a qualidade e com o tipo de apresentação dos resultados da pesquisa de AC. Enquanto a qualidade da apresentação demonstra uma postura comercial adequada, o tipo de apresentação pode facilitar a compreensão dos resultados, por parte do contratante. Em função das características dos resultados de uma pesquisa de $\mathrm{AC}$, o pesquisador deve se expressar de forma simples, utilizando recursos descritivos e principalmente gráficos. Desta forma, ao ler o relatório, o contratante terá maior facilidade em
Esta pesquisa foi conduzida de forma a ilustrar o uso de Análise Conjunta com Estimulação em Duas Etapas (AC com E2E). Ela se refere a uma pesquisa sobre o mestrado profissionalizante da Escola de Engenharia da Universidade Federal do Rio Grande do Sul (UFRGS), apresentada em Battesini (2002). A Escola de Engenharia oferece o Curso de Mestrado Profissionalizante (MP) no município de Porto Alegre - RS, que tem duração de 18 meses e é dividido em duas etapas. No período inicial de 12 meses, são desenvolvidas as disciplinas e o período final é dedicado ao desenvolvimento da dissertação.

A Escola de Engenharia pretendia definir uma estratégia de marketing para seu MP que direcionasse as suas ações e investimentos, de forma a manter os seus índices de aceitação e a consolidar sua condição de liderança no ensino e pesquisa. Neste contexto, o problema de marketing estabelecido é a definição da estratégia a ser adotada em relação ao perfil dos cursos ofertados

\subsection{Identificar o problema e definir os objetivos da pesquisa}

A partir do problema de marketing, foi estabelecido o problema de pesquisa, que é determinar os motivos que levam os alunos a escolher o MP ofertado pela Escola de Engenharia. A AC com E2E foi escolhida como técnica de pesquisa por propiciar a obtenção das informações, de forma indireta em um ambiente de competição entre cursos (cenários hipotéticos). O objetivo da pesquisa foi identificar, em relação aos motivos que levam os alunos a preferir o curso de MP oferecido pela Escola de Engenharia: a influência dos atributos determinantes, suas importâncias relativas, bem como a existência de interações entre atributos.

\subsection{Desenvolver o plano de pesquisa}

\subsubsection{Definir os atributos, os níveis dos atributos} e o relacionamento das Part-Worth

A escolha dos atributos para a pesquisa de AC com E2E foi realizada através de uma etapa qualitativa. A pesquisa qualitativa teve o objetivo de identificar os atributos importantes na decisão de cursar o mestrado profissionalizante da escola de Engenharia. Nesta etapa, foram 
entrevistados 70 alunos. Os sete atributos selecionados possuem apenas dois níveis e são apresentados na Figura 5, em seus níveis reais e codificados (NC).

Para tornar a avaliação do respondente o mais real possível, o preço e as condições de pagamento dos diversos cenários foram apresentados juntamente com os demais atributos. Desta forma, para cada curso hipotético (cenário), foi apresentado o valor real associado (R\$) e o número de parcelas, conforme a sua composição de níveis dos atributos. Por questões estratégicas, não será apresentada a sistemática utilizada na composição dos custos dos cenários apresentados aos respondentes. Como todos os atributos possuem apenas dois níveis, as Part-Worth ficaram condicionadas a um relacionamento Linear.

\subsubsection{Definir a regra de composição}

Foi adotada a regra de composição aditiva com interações por dois motivos: em função dos respondentes conhecerem o objeto de pesquisa e da suspeita da existência de interações entre atributos, o que propicia a ocorrência de efeitos de interações.

\subsubsection{Definir a estimulação da coleta de dados}

\subsubsection{Definir a combinação dos atributos para a composição dos cenários}

O número total de combinações entre atributos corresponde a $2^{7}=128$ cenários. Em função do grande número de combinações foi utilizado um planejamento experimental do tipo $2^{\mathrm{k}}$ fracionado duas vezes $\left(2^{7-2}\right)$. O fracionamento consistiu em agrupar os cenários em quatro frações ortogonais, cada uma delas contendo 32 cenários, sendo que apenas uma fração (Fração 1) foi apresentada para avaliação dos respondentes.

O procedimento de fracionamento impôs a perda da capacidade de avaliar, de forma independente, o efeito de duas interações de quinta ordem (ABCDE e CDEFG) utilizadas como Contrastes de Definição, e além delas, o efeito de uma interação de quarta ordem (ABFG). Outro inconveniente do fracionamento é o vínculo entre os efeitos, desta forma, não é possível atribuir a causa de determinado efeito a um único atributo ou interação de atributos. Outro inconveniente do procedimento de fracionamento foi o vínculo de seis interações de segunda ordem, de um total de 21 efeitos de terceira ordem. Os efeitos de interação de segunda ordem FG, AF e AG foram vinculados respectivamente à $\mathrm{AB}, \mathrm{BG}$ e $\mathrm{BF}$. Os atributos Ênfase, Título Obtido e Entidade, foram atribuídos respectivamente aos efeitos $\mathrm{B}, \mathrm{F}$ e G, uma vez que estimase que os mesmos têm menor probabilidade de interagir entre si.

Desta forma, os 31 efeitos a serem estimados são sete de primeira ordem (A, B, C, D, E, F, G), 18 de segunda ordem (FG, AC, AD, AE, AF, AG, BC, BD, BE, CD, CE, CF, CG, DE, DF, DG, EF, EG) e seis de terceira ordem (ACF, ACG,ADF,ADG,AEF,AEG).

Após o procedimento de fracionamento, os cenários da Fração 1 foram blocados três vezes. Desta forma, no procediemnto de blocagem, os 32 cenários da matriz experimental foram agrupados em oito blocos ortogonais, cada um contendo quatro cenários. As sucessivas blocagens impuseram a perda da capacidade de avaliar de forma independente o efeito de três interações de quarta ordem (ACEF, ADEF e BCDG), utilizadas como contraste de definição. Além deles, ficaram confundidos com o efeito dos blocos, dois efeitos de interação de segunda ordem e dois de sexta ordem, respectivamente $\mathrm{CD}$ e $\mathrm{BG}$ (vinculado à $\mathrm{AF}$ ), $\mathrm{ABDEFG}$ e ABCEFG. O procedimento de blocagem permitiu criar conjuntos competitivos de cenários e reduzir a uma quantidade aceitável o número de cenários simultaneamente avaliados pelos respondentes em cada bloco (correspondentes à primeira etapa da estimulação). Desta forma, através da blocagem, cada respondente

\begin{tabular}{|c|c|c|c|c|c|}
\hline \multirow{2}{*}{\multicolumn{2}{|c|}{ Atributos }} & \multicolumn{4}{|c|}{ Níveis dos Atributos } \\
\hline & & Reais & NC & Reais & NC \\
\hline A & Horário & $\begin{array}{l}\text { Aulas durante a Semana } \\
\left(3^{\underline{a}}, 4^{\underline{a}} \text { e } 5^{\underline{a}} \text { feira à noite }\right)\end{array}$ & -1 & $\begin{array}{l}\text { Aulas no fim de Semana (6 } 6^{a} \text { à tarde } \\
\text { e à noite) e Sábado (manhã) }\end{array}$ & 1 \\
\hline B & Disciplina/ênfase & Currículo fixo de disciplinas & -1 & $\begin{array}{l}\text { Currículo com escolha flexível das } \\
\text { disciplinas, adaptado a cada aluno }\end{array}$ & 1 \\
\hline C & Local & $\begin{array}{l}\text { Aulas em bairros afastados } \\
\text { (com estacionamento) }\end{array}$ & -1 & Aulas no Centro (sem estacionamento) & 1 \\
\hline D & $\mathrm{N}^{\circ}$ Alunos por sala & 15 Alunos/Sala de aula & -1 & 30 Alunos/Sala de aula & 1 \\
\hline E & Enfoque do Curso & Pós-Graduação com enfoque Acadêmico & -1 & Pós-Graduação com enfoque Profissional & 1 \\
\hline $\mathbf{F}$ & Título Obtido & Título de Especialista (360h/aula) & -1 & Título de Mestre (450h/aula) & 1 \\
\hline G & Entidade & Não ofertado pela UFRGS & -1 & Ofertado pela UFRGS & 1 \\
\hline
\end{tabular}

Figura 5 - Atributos selecionados e seus níveis 
precisou avaliar apenas quatro blocos (16 cenários), ao invés dos oito blocos (32 cenários).

Assim, após os procedimentos de fracionamento e blocagem cada respondente recebeu quatro cartões (blocos) contendo quatro cenários cada um, sendo necessário dois respondentes para a avaliação de todos cenários da Fração 1. Enquanto um respondente avaliou os cartões A, B, C e D (bloco par), o outro avaliou os cartões E, F, Ge H (bloco ímpar). A Figura 6 apresenta a composição de cenários (codificados) para cada cartão.

\subsubsection{Definir a escala de medida da variável de resposta}

Tanto os respondentes da amostra, quanto os da amostra de validação (ver item 3.2.4) foram estimulados a realizar a avaliação dos cenários em duas etapas. Na primeira etapa da estimulação, os respondentes (amostra e amostra de validação) apenas escolheram um cenário dos cenários de cada cartão, o que simplificou sua tarefa permitindo assim que eles adotassem nesta etapa um modelo de escolha não-compensatório. Na segunda etapa da estimulação, os respondentes da amostra realizaram avaliações utilizando uma escala Intervalar de 0 a 100 pontos, sendo que os respondentes puderam atribuir uma mesma quantidade de pontos a mais de um cenário. Enquanto que, para facilitar a sua tarefa, os respondentes da amostra de validação apenas escolheram (escala nominal binária) um dos cenários previamente selecionados na primeira etapa da estimulação. Assim, na segunda etapa, em função do menor número de cenários, os respondentes puderam realizar uma avaliação mais refinada e utilizar um modelo de escolha compensatório, ou até mesmo manter aquele não-compensatório.

\subsubsection{Definir a forma de representação dos cenários}

Os cenários foram representados nos oito cartões através de descrições textuais. Os cartões foram identificados através de uma letra maiúscula (de $\mathrm{A}$ a $\mathrm{H}$ ), sendo que em cada cartão foram apresentados quatro cenários. Os cenários de cada cartão foram identificados através de um número (1 a 4), desta forma, C1 identifica o primeiro cenário apresentado no cartão $\mathrm{C}$, do seu topo para baixo.

Em todos os cenários, os atributos foram apresentados na mesma ordem a partir do topo do cartão. Este procedimento foi adotado para facilitar as avaliações dos respondentes, que possuíam uma boa compreensão do serviço objeto de pesquisa, e desta forma, presumiu-se que a ordem de apresentação dos atributos não influenciaria os resultados.

\subsubsection{Determinar plano de amostragem e o nível de análise}

A população a ser investigada era composta por 150 alunos do mestrado profissionalizante oferecido pela Escola de Engenharia, que estavam cursando o primeiro ano da turma 2001/2002. Desta forma, a população investigada é composta por indivíduos que já haviam realizado a opção pelo mestrado profissionalizante e, portanto, conheciam o serviço objeto da pesquisa.

O curso oferece aos alunos seis áreas de concentração: Gerência de Serviços (GS), Gerência de Produção(GP), Qualidade e Desenvolvimento de Produto e Processo (QDPP), Ergonomia (ERG), Logística (LOG) e Transportes (TRAN), sendo que cada área de concentração foi considerada um estrato. Em função da facilidade de acesso aos indivíduos da população para cada estrato, a proposta foi de pesquisar o maior número possível de alunos. A Tabela 1 apresenta a composição de alunos da população (150 alunos) e da amostra (51 alunos, ou seja 34\%).

Além da amostra de 51 alunos, a pesquisa foi aplicada a uma amostra de validação constituída por 44 alunos. Desta forma, foram entrevistados 95 alunos $(51+44)$, ou

\begin{tabular}{|c|c|c|c|c|c|c|c|c|c|c|c|}
\hline \multicolumn{12}{|c|}{ Bloco Ímpar } \\
\hline \multicolumn{3}{|c|}{ Cartão A } & \multicolumn{3}{|c|}{ Cartão B } & \multicolumn{3}{|c|}{ Cartão C } & \multicolumn{3}{|c|}{ Cartão D } \\
\hline 1 & A1 & abcde & 5 & B1 & c & 9 & C1 & $d$ & 13 & D1 & abe \\
\hline 2 & A2 & bf & 6 & B2 & adef & 10 & C2 & acef & 14 & D2 & bcdf \\
\hline 3 & A3 & $\mathrm{ag}$ & 7 & B3 & bdeg & 11 & C3 & bceg & 15 & D3 & acdg \\
\hline 4 & A4 & cdefg & 8 & B4 & abcfg & 12 & C4 & abdfg & 16 & D4 & efg \\
\hline \multicolumn{12}{|c|}{ Bloco Par } \\
\hline \multicolumn{3}{|c|}{ Cartão E } & \multicolumn{3}{|c|}{ Cartão F } & \multicolumn{3}{|c|}{ Cartão G } & \multicolumn{3}{|c|}{ Cartão H } \\
\hline 17 & E1 & e & 21 & F1 & abd & 25 & G1 & $a b c$ & 29 & H1 & cde \\
\hline 18 & E2 & acdf & 22 & F2 & bcef & 26 & G2 & bdef & 30 & H2 & af \\
\hline 19 & E3 & bcdg & 23 & F3 & aceg & 27 & G3 & adeg & 31 & H3 & bg \\
\hline 20 & E4 & abefg & 24 & F4 & dfg & 28 & G4 & $\mathrm{cfg}$ & 32 & H4 & abcdefg \\
\hline
\end{tabular}

Figura 6 - Composição de cenários para cada cartão 
cada uma, um cartão com quatrocenários (ver Figura 7). Para que não houvesse constrangimento dos alunos, não foi solicitada a sua identificação pessoal.

\subsection{Coletar as informações}

Tanto na etapa qualitativa quanto na pesquisa de AC com E2E, a coleta de dados foi realizada nas salas de aula freqüentadas pelos alunos. Não foram constatadas dificuldades em relação ao manuseio dos questionários, apesar dos entrevistadores terem sido solicitados a elucidar dúvidas de alguns respondentes. Atribui-se estas dúvidas ao fato de a $\mathrm{AC}$ com E2E não ser um tipo usual de pesquisa. As turmas (disciplinas) eram compostas por 20 a 30 alunos de variadas ênfases, o que demandou um trabalho de planejamento, de forma a obter um número equilibrado de respondentes, entre a amostra e a amostra de validação, e entre o bloco par e ímpar de cenários. O total de 95 entrevistas foi realizado em dois finais de semana sucessivos, sendo que o tempo médio de entrevista por sala de aula foi de 30 minutos, entre a explanação inicial e o recolhimento dos questionários.

\subsection{Analisar as informações}

\subsubsection{Definir o tipo de análise}

Inicialmente, foi realizado um estudo do comportamento das avaliações formalmente realizadas na segunda etapa da estimulação. A Figura 8 apresenta a dispersão dos dados para cada cenário (1 a 32), onde pode-se observar um comportamento compatível com a condição de homocedasticidade da variância.

Em função do tipo de dado obtido na coleta (escala intervalar), a modelagem se deu através da Análise de Regressão Linear Múltipla com o auxílio do software estatístico SPSS. Desta forma, foi gerado um modelo de Preferências (Y) agregado geral para todos os respondentes da amostra (ver Equação 4). O modelo gerado

\begin{tabular}{|c|c|c|c|c|}
\hline & Cenário A1 & Cenário A2 & Cenário A3 & Cenário A4 \\
\hline \multirow{8}{*}{ 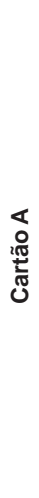 } & Título de Especialista (360h/aula) & Título de Mestre (450h/aula) & Título de Especialista (360h/aula) & Título de Mestre (450h/aula) \\
\hline & Não ofertado pela UFRGS & Não ofertado pela UFRGS & Ofertado pela UFRGS & Ofertado pela UFRGS \\
\hline & Enfoque profissional & Enfoque acadêmico & Enfoque acadêmico & Enfoque profissional \\
\hline & $\begin{array}{l}\text { Currículo com escolha flexível } \\
\text { das disciplinas }\end{array}$ & $\begin{array}{l}\text { Currículo com escolha flexível } \\
\text { das disciplinas }\end{array}$ & Currículo fixo de disciplinas & Currículo fixo de disciplinas \\
\hline & 30 alunos por sala de aula & 15 alunos por sala de aula & 15 alunos por sala de aula & 30 alunos por sala de aula \\
\hline & $\begin{array}{c}\text { Aulas no Centro } \\
\text { (sem estacionamento) }\end{array}$ & $\begin{array}{c}\text { Aulas em bairros afastados (com } \\
\text { estacionamento) }\end{array}$ & $\begin{array}{l}\text { Aulas em bairros afastados } \\
\text { (com estacionamento) }\end{array}$ & $\begin{array}{c}\text { Aulas no Centro } \\
\text { (sem estacionamento) }\end{array}$ \\
\hline & $\begin{array}{c}\text { Aulas no fim de semana } \\
\text { (6 } 6^{\mathrm{a}} \text { à tarde e à noite }+ \\
\text { Sábado pela manhã) }\end{array}$ & $\begin{array}{l}\text { Aulas durante a semana } \\
\quad\left(3^{\mathrm{a}}, 4^{\mathrm{a}}, 5^{\mathrm{a}} \text { à noite }\right)\end{array}$ & $\begin{array}{l}\text { Aulas no fim de semana } \\
\text { (6 } 6^{\mathrm{a}} \text { à tarde e à noite }+ \\
\text { Sábado pela manhã) }\end{array}$ & $\begin{array}{l}\text { Aulas durante a semana } \\
\quad\left(3^{\mathrm{a}}, 4^{\mathrm{a}}, 5^{\mathrm{a}} \text { à noite }\right)\end{array}$ \\
\hline & 12 parcelas de $\mathrm{R} \$ 510 /$ mês & 24 parcelas de $\mathrm{R} \$ 720 /$ mês & 12 parcelas de $R \$ 590 /$ mês & 24 parcelas de $R \$ 640 /$ mês \\
\hline
\end{tabular}

Figura 7 - Cartão A com os cenários em seus níveis reais 
Tabela 2 - Valores dos Coeficientes, efeitos e das importâncias relativas 
Figura 9 - Gráfico de dispersão dos Resíduos padronizados

\subsubsection{Avaliar a confiabilidade e validade dos resultados}

A presença de termos de interação no modelo (Equação 4) valida a regra de composição adotada (aditiva com interações), ou seja, a suposição da existência de termos de interação foi confirmada pelo modelo.

Os valores de $\mathrm{R}^{2}=0,247$ e de $\mathrm{R}_{\text {ajustado }}^{2}=0,238$ referentes ao modelo apresentado na Equação 6 indicariam uma baixa capacidade $(24,7 \%$ ou $23,8 \%)$ de explicar a preferência dos alunos. A seguir é apresentada a abordagem proposta em AC com E2E para verificar o adequado ajuste do modelo que é a utilização do valor de $\left(\mathrm{R}^{2} / \mathrm{R}^{2}{ }_{\text {máx }}\right)$ conjuntamente com a capacidade de prever as melhores respostas (percentual de acertos dos cenários preferidos).

$\mathrm{O}$ valor de $\mathrm{R}_{\text {máx }}^{2}=0,256$ pode ser calculado através da Equação 4, a partir dos valores de $\mathrm{SQT}=830045,41 \mathrm{e}$ do cálculo do valor de SQEP = 617589,64, através da
Equação 5. A estatística $\mathrm{R}_{\text {máx }}^{2}$ leva em consideração somente o erro devido à falta de ajuste do modelo, sem considerar a variabilidade devido ao erro puro, ou seja, entre as repetições das respostas atribuídas por diferentes respondentes a um mesmo cenário. Desta forma, o valor de $R^{2} / R_{\text {máx }}^{2}=0,247 / 0,256=0,9636$, indica que o modelo consegue representar $96,36 \%$ da variabilidade que pode ser modelada por algum modelo de regressão, o que indica um adequado ajuste do modelo aos dados.

A validade do modelo da Equação 4 foi avaliada pela sua capacidade de prever as melhores escolhas para a amostra e para a amostra de validação. Para a amostra, foi verificado o percentual de vezes que o modelo foi capaz de prever os cenários preferidos pelos respondentes $\left(1^{\mathrm{a}}, 2^{\mathrm{a}}, 3^{\mathrm{a}}\right.$ e $4^{\mathrm{a}}$, onde $1^{\mathrm{a}}=$ cenário que recebeu a maior pontuação). Já para a amostra de validação, foi verificado o percentual de vezes que o modelo foi capaz de prever o cenário preferido $\left(1^{\mathrm{a}}\right)$. Esta análise indicou que o modelo foi capaz de prever $45,10 \%$ das vezes que o cenário com melhor avaliação ( $1^{\text {a }}$ posição) e $31,37 \%$ vezes o cenário com a segunda melhor avaliação, ou seja, o modelo conseguiu prever $76,47 \%$ das vezes, pelo menos um dos dois cenários preferidos pelos respondentes. Em relação à amostra de validação, foram encontrados percentuais semelhantes, sendo que o modelo foi capaz de prever $43,18 \%$ das vezes o cenário escolhido $\left(1^{\mathrm{a}}\right)$.

Em função da adequada capacidade do modelo e da capacidade de previsão do modelo, é possível afirmar que o modelo apresentado na Equação 4 possui uma adequada capacidade de previsão das preferências dos consumidores, diferentemente do que indicava o valor de $\mathrm{R}^{2}$.

\subsubsection{Realizar simulações}

Uma vez identificado um modelo válido, foram realizadas simulações para investigar a preferência dos respondentes em relação aos 96 cenários não apresentados e para avaliar o comportamento do modelo em relação à inserção de variáveis de segmentação (estratos).

Pela Equação 4, foram calculados os Valores Previstos (VP) para os 128 cenários possíveis. Esta investigação teve como objetivo identificar cenários com valores de preferência similares àqueles que receberam melhores avaliações entre os que foram submetidos aos respondentes (Fração 1). A Tabela 6 apresenta os valores previstos para cada um dos 128 cenários (C) ordenados pelos seus respectivos VP, sendo que os cenários hachurados correspondem àqueles da Fração 1.

Como pode ser observado na Tabela 3, os cenários abefg, abcdefg, abcefg e abdefg são os que possuem melhor avaliação, sendo que dentre estes apenas um pertencia à Fração 1. A indentificação de três cenários 
Figura 10 - Gráfico das Importâncias Relativas dos atributos do modelo

Desta forma, qualquer que seja a estratégia utilizada na solução do problema de marketing, os atributos A, E, F e $\mathrm{G}$ devem merecer destaque. Esta afirmação é corroborada pelos maiores valores previstos $(\mathrm{VP}=57,5)$ estarem associados aos cenários abefg, abcdefg, abcefg e abdefg.

\section{Conclusão}

Este artigo apresentou um método de AC com estimulação em Duas Etapas (AC com E2E). O método proposto incorpora a perspectiva do respondente através da estimulação da coleta de dados em duas etapas, que 
permite reduzir o número de avaliações formalmente realizadas. O método utiliza as cinco macro-etapas de uma pesquisa de marketing, propostas por Kotler (2000), e as etapas de um planejamento experimental, propostas por Montgomery (1976). O objetivo desta abordagem é integrar as etapas já consolidadas para uma pesquisa de marketing com as etapas de um planejamento experimental, gerando um método também consistente com os diversos métodos de $\mathrm{AC}$ apresentados na literatura. Assim, a AC com E2E, ao invés de propor inúmeras avaliações por parte dos respondentes, de modo a satisfazer conceitos estatísticos, propõe a simplificação da coleta de dados ao considerar a capacidade de avaliação dos respondentes (modelos de decisão utilizados). Desta forma, o respondente não precisa alterar substancialmente seu comportamento de compra ao responder a pesquisa de AC.

O planejamento de uma pesquisa de $\mathrm{AC}$ com E2E baseia-se: na sua estruturação de suas de etapas, na estimulação da coleta de dados em duas etapas, no uso da blocagem, na redução do número de avaliações formalmente realizadas pelo respondente utilizando uma escala de medida (em relação ao total de cenários planejados experimentalmente) e na modelagem das preferências a partir apenas das avaliações formalmente realizadas na segunda etapa da estimulação.

Para ilustrar o uso da AC com E2E foi conduzida uma pesquisa sobre o Mestrado Profissionalizante oferecido pela Escola de Engenharia da Universidade Federal do Rio Grande do Sul. Esta aplicação da AC com E2E permitiu a geração de um modelo com adequada capacidade de previsão das preferências dos consumidores, onde foi determinada a influência dos atributos importantes, suas importâncias relativas, bem como a existência de interações entre eles. Os atributos que influenciam significativamente a preferência dos alunos são o Título Obtido (F), o Enfoque do Curso (E), a Entidade (G), a Disciplina/Ênfase (B) e o Horário (A). Os atributos Local (C) e Número de alunos por sala (D) não apresentaram efeitos significativos. Foram identificados quatro cursos (cenários) promissores: abefg, abcdefg, abcefg e abdefg. Estes cursos correspondem àqueles que ofertam Aulas no fim de semana ( $6^{\mathrm{a}}$ à tarde e à noite e sábado de manhã), Currículo com escolha flexível das disciplinas adaptada a cada aluno, Enfoque Profissional, Título de Mestre e que fossem ofertados pela UFRGS, e independentemente do nível ofertado: Aulas em bairros afastados (com estacionamento) ou Aulas no centro (sem estacionamento) e 15 ou 30 alunos por sala de aula.

Desta forma, a AC com E2E pode ser considerada uma variante da Análise Conjunta Tradicional e da ChoiceBased Conjoint Analysis (CBCA), onde os julgamentos de preferência do consumidor são modelados através de técnicas de decomposição, com base em avaliações de cenários Full Profile obtidos a partir de uma forma indireta e em um ambiente de competição entre grupos de cenários (Choice Set). A AC com E2E se diferencia da Traditional Conjoint Analysis (TCA), ao propor a flexibilidade no uso da escala (métrica e nominal binária) e a avaliação formal apenas de um número menor de cenários, e também da Choice-Based Conjoint Analysis (CBCA), ao compor todos os Choice Set com o mesmo número de cenários e sem a opção no choice.

\section{Referências}

BATTESINI, M.; CATEN, C. S. T. Uso de Análise Conjunta no Desenvolvimento de Produtos. In: Congresso Brasileiro de Gestão e Desenvolvimento de Produtos, 3., 2001, Florianópolis.

BOYD, H.W.; WESTFALL, H.; STATSCH, S. F. Marketing Research: text and cases. Irwin: Homewood IL, 1989.

BURNS, A. C.; BUSH, R. F. Marketing Research. New Jersey: Prentice Hall, 1995.

CATEN, C. S. T.; BATTESINI, M. Análise Conjunta: Uma abordagem para obtenção de maiores informações sobre o comportamento do consumidor. In: Encontro Nacional de Engenharia de Produção, 21., 2001, Salvador.

CHURCHILL Jr., G. A.; NIELSEN Jr., A. C. Marketing Research: Methodological Foundations. 6. ed. Wisconsin: The Dryden Press, 1996.

EVRARD, Y.; PRAS, B.; ROUX, E. Market Estudes et recherches en marketing - Foundements Méthodes. 1. ed. Paris: Editions Nathan, 1993. 629p.

GREEN, P. E.; KRIEGER, A. M. Segmenting Markets with Conjoint Analisys. Journal of Marketing, v. 55, p. 20-31, October, 1991.

GREEN, P. E.; SRINIVASAN, V. Conjoint Analisys in Marketing: New Developments With Implications for Research and Practice. Journal of Marketing, v. 54, p. 3-19, Out. 1990.

GUSTAFSSON,A.; EKDAHL, F.; BERGMAN, B. Conjoint analysis: A useful tool in the design process. Total Quality Management, Abngdon, May 1999. 15p. Disponível em: http://www.proquest.com.br, arquivo capturado em $17 / 5 / 2000$.

HAIR Jr., J. F.; ANDERSON, R. E.; TATHAM, R. L.; BLACK, W. C. Multivariate Data Analysis. New Jersey: Prentice-Hall Inc., 1998. 730p. 
INTELLIQUEST - INFORMATION SOLUTIONS FOR GLOBAL TECHNOLOGY MARKETING. Preference Structure Measurement: Conjoint Analysis and Related Techiniques - A Guide dor Designing and Interpreting Conjoint Studies. 2.ed. Austin: American Marketing Association, s.d. 29p. (Marketing Research Techniques Series).

KOTLER, P. Marketing para o século XXI. 6. ed. São Paulo: Futura, 1999. 305p.

KOTLER, P. Administração de Marketing: a edição do novo milênio. $10^{\mathrm{a}}$ ed. $2^{\mathrm{a}}$ reimpressão. São Paulo: Prentice Hall, 2000. Cap. 4, p. 121-140 e Cap 11, p. 349-363.

LEE, J.; GEISTFELD, L. V. Enhancing consumer choice: Are we making appropriate recommendations? The Journal of Consumer Affairs, Madison, Winter 1998. Disponível em: http://www.proquest.com.br. Arquivo capturado em 17/5/2000.

MALHOTRA, N. K. Pesquisa de Marketing: Uma Orientação Aplicada. 3. ed. Porto Alegre: Bookman, 2001.719p.

MILLER, J. T.; OGDEN, J. R.; LATSHAW, C. A. Using trade-off analysis to determine value-price sensitivity of custom calling. Jan 1998. Disponível em: http:// www.proquest.com.br, arquivo captu-rado em 17/05/ 2000.

MONTGOMERY, D. C. Design and analisys of experiments. 3. ed. New York: John Wiley \& Sons, 1976. 533 p.

MONTGOMERY, D. C.; PECK, E. Introduction to Linear Regression Analysis. 2. ed. New York: WileyInterscience, 1992.

SHETH, J. N.; MITTAL, B.; NEWMAN, B. I. Costumer Behavior. Fort Worth: The Dryden Press, 1999.

WERKEMA, M. C. C.; AGUIAR, S. Análise de Regressão: Como entender o relacionamento entre as variáveis de um processo. Belo Horizonte: Fundação Christiano Ottoni, 1996. v.7. 311p. (Série Ferramentas da Qualidade).(a)

YIN, R. K. Case Study Research:design and methods. 2. ed. Thousand Oaks: SAGE Publications,1994.171p. 
